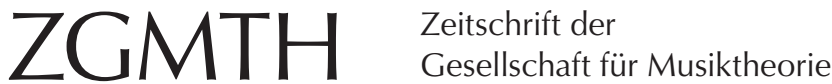

Huschner, Roland (2017): Zur produktionsbezogenen Perspektive bei der Analyse von Popmusik. ZGMTH 14/1, 161-187. https://doi.org/10.31751/900

(C) 2017 Roland Huschner (huschner@gmail.com)

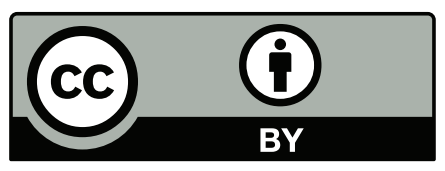

Dieser Text erscheint im Open Access und ist lizenziert unter einer Creative Commons Namensnennung 4.0 International Lizenz.

This is an open access article licensed under a Creative Commons Attribution 4.0 International License.

eingereicht / submitted: 28/02/2017

angenommen / accepted: 23/03/2017

veröffentlicht / first published: 30/06/2017

zuletzt geändert / last updated: 13/09/2018 


\title{
Zur produktionsbezogenen Perspektive bei der Analyse von Popmusik
}

\author{
Roland Huschner
}

\begin{abstract}
Das Tonstudio ist in der Popmusik der Ort, an dem die wichtigsten musikalischen Entscheidungen getroffen werden. Dort erhalten die Songs oder Tracks im Rahmen von Verhandlungen zwischen Studioakteur*innen und Musiker*innen jene musikwissenschaftlich schwer fassbare klangliche 'Konkurrenzfähigkeit` und Form, die sie musikalisch und außermusikalisch sanschlussfähig`machen sollen. In Analysen, die sich ausschließlich auf die Klangkonfigurationen von Popmusik konzentrieren, entfällt die Untersuchung dieser klangstrukturierenden soziokulturellen Prozesse innerhalb von Tonstudios und deren potentieller Auswirkungen auf die Rezeption. Der vorliegende Aufsatz zeigt anhand ausgewählter Beispiele einer ethnographischen Studie den möglichen Mehrwehrt einer sowohl handwerklich als auch soziokulturell fundierten Produktionsperspektive für analytische Zugriffe auf populäre Musik.
\end{abstract}

In pop music, the recording studio is the place where the most important musical decisions are made. It is there that songs or tracks, in the course of negotiations between producers, technicians and musicians, achieve that 'competitiveness' and form which are supposed to make them musically and metamusically 'accessible', features which are not easy to grasp musicologically. Analyses that focus exclusively on the sound configurations of pop music tend to ignore the sociocultural processes structuring the song and its sound as well as their potential effect on the song's reception. Based on selected examples taken from an ethnographic study, this article reveals the advantages of a technically as well as socioculturally informed production perspective for the analysis of popular music.

Bereits vor zwanzig Jahren stellte Simon Frith fest, dass das Tonstudio der Ort sei, »where the most interesting and influential musical value judgements are made. « Weiterhin wies er in diesem Zusammenhang auf die Bedeutung der in den Tonstudios wirkenden Tontechniker*innen und vor allem Produzent*innen hin, die entgegen einer häufigen Wahrnehmung die Kommunikation zwischen Künstler*innen und Hörenden nicht stören oder verhindern, sondern vielmehr unterstützend wirken würden: „they may, in fact, make that communication possible. $\aleph^{2}$ Frith untersuchte die sich grundlegend verändernde Wahrnehmung der Künstler- und Produzent*innenrolle innerhalb der Rockmusik der späten 1960er Jahre und kam zum Ergebnis, dass der Einfluss der Produzent*innen am Ende eines mehrjährigen Wandlungsprozesses durch Musiker*innen und Journalist*innen

1 Frith 1998, 59.

2 Ebd., 62. 
vor allem als nachteilig angesehen wurde. ${ }^{3}$ Die Dauerhaftigkeit solcher Wertungen thematisiert Peter Doyle 2009 in seinem Podcast Working for the Man: Die (nichtakademische) Darstellung der Beziehung zwischen Künstler*innen und Produzent*innen falle häufig zum Nachteil der letztgenannten aus und beschreibe diese z.B. als »unrepentant corrupter of artistic purity. ${ }^{4}$ Bei Allan Moore heißt es deutlich neutraler: »Producers and engineers [...] alter the sound that they hear in order to create the sound they believe listeners will accept.$^{5}$ Allerdings spielt auch in Moores Überlegungen die Idee des Veränderns der Klangkonfigurationen ohne großes Mitspracherecht der betroffenen Musiker*innen eine Rolle.

Es ist u.a. zur Aufklärung solcher Annahmen begrüßenswert, dass der besonderen Rolle und Funktion von Tonstudios (samt den dort tätigen Akteur*innen) in den Prozessen der populären Musikproduktion in den letzten zehn Jahren - zuvor geschah das nur sporadisch $^{6}$ - verstärkte Aufmerksamkeit zuteilwurde. ${ }^{7}$ Bisher wurden Tonstudios zumeist als eine Art sblack box` wahrgenommen, aus welcher Bands oder Einzelmusiker*innen nach einem mehr oder weniger langen Zeitraum mit einem Tonträger herauskamen, der die dort aufgenommenen oder entstandenen Klangkonfigurationen enthielt. Gleichzeitig scheint es aber bei den die Tonstudios nutzenden Musiker*innen spezifische und zugleich sich stark voneinander unterscheidende Vorstellungen davon zu geben, was im Tonstudio passiert bzw. zu passieren hat ${ }^{8}$, ohne dass die Institution Tonstudio und die damit verbundene Rolle der Produzent*innen hinterfragt würden: Entweder wird in Abhängigkeit von den verfügbaren Mitteln ein möglichst professionelles Tonstudio bzw. Projektstudio samt dazugehörigen Akteur*innen gemietet oder es wird in Ermangelung von Ressourcen im Homestudio sselbst produziertı. Der Schritt der Produktion wird, in welcher Konstellation auch immer, nie übersprungen. Was dieser Schritt indes konkret beinhaltet, kann weder begrifflich genau bestimmt noch antizipiert werden und ergibt sich häufig erst im Verlauf der Produktionsprozesse. ${ }^{9}$ Tonstudios sind in Form der Produktion und des Masterings also jene Orte, die den Klangkonfigurationen der Popmusik ihre für eine Publikation finale Erscheinungsform geben. ${ }^{10}$ Trotz der daraus folgenden

3 Frith 2012, zitiert nach: Zagorski-Thomas 2014, 207.

4 Doyle 2009.

5 Moore 2012, 188.

6 Vgl. u.a. Massey 2000; Zak 2001; Meintjes 2003; Moehn 2005.

7 Vgl. u.a. das Journal on the Art of Record Production (JARP), http://arpjournal.com (28.2.2017); Burgess 2005; Zagorski-Thomas 2014, 108; Huschner 2016.

8 Kramarz 2014, 57; Thompson/Lashua 2016.

9 Huschner 2016, 6-30, 355-370; Moore 2012, 188.

10 Mit sKlangkonfiguration sche, analoge oder natürliche Klänge erzeugen, bearbeiten und konfigurieren, wodurch am Ende dieser Vorgänge eine Auswahl und Anordnung von Klängen als Resultat steht, die wir beim Hören dechiffrieren, also nach uns bekannten Mustern zusammensetzen. Nicht jede Studioproduktion zielt automatisch auf massenhaften Konsum ab (z.B. ein Song als Geburtstagsgeschenk). Ich gehe hier dennoch vorrangig von Aktivitäten aus, die mittels getätigten Investitionen zu einer Positionierung im Feld der Popmusik führen und nach Möglichkeit Profite erwirtschaften sollen. Vgl. Huschner 2016, 59, 269-272; zur Kritik an der Unterscheidung zwischen »kommerziell « und »nicht-kommerziell« vgl. Wicke 1993. >(Popmusik-)Publikation bezeichnet - bezogen auf Songs, Tracks usw. - die 
Relevanz dieser Prozesse beschränkten sich Betrachtungen von Tonstudios bisher meist auf historische und technische Aspekte. ${ }^{11}$ Beschreibungen der Interaktionen zwischen Produzent*innen, Tonmeister*innen und Popmusiker*innen liegen in Form von einigen (älteren) ethnographischen Studien ${ }^{12}$ vor, deren Erkenntnisinteresse sich jedoch nicht explizit auf das Erklären dieser Interaktionen und ihrer Auswirkungen bezog. ${ }^{13}$

Mein Anliegen war es daher, die sblack box` Tonstudio zu erhellen. Im Rahmen meiner Forschungen besuchte ich über zwei Jahre hinweg Sessions in verschiedenen Berliner Studios. Die von mir beobachteten Bands würde ich allgemein den Genres Rock, Jazz, Pop und Deutscher Schlager zuordnen. Die Bandbreite der beobachteten Sessions reichte vom Einspielprozess über das Editing und Mixen bis hin zum Mastering; die Studios wiederum variierten vom Wohnzimmer mit Gesangskabine bis hin zu Studios, die bezüglich des finanziellen Aufwandes ihrer Ausstattung und der Gebäudekosten deutlich im sechs- oder sogar siebenstelligen Bereich zu verorten waren. In solchen hochpreisigen Studios wurden dementsprechend Aufnahmen und/oder Mischungen für national oder international erfolgreiche Formationen angefertigt. Meine Rolle in den jeweiligen Sessions variierte zwischen teilnehmendem Beobachter, Sessionmusiker, Co-Produzent, Bandmitglied oder kompositorischem Ideengeber. Bei den Sessions, die zwischen vier und mehr als acht Stunden dauerten, wurde mir gestattet, ein Diktiergerät mitlaufen zu lassen, sodass Gespräche der für die Analyse ausgewählten Sessions anschließend transkribiert werden konnten. ${ }^{14}$ Die Analyse der Transkriptionen, welche u.a. in Auseinandersetzung mit Pierre Bourdieus Kapitalsorten und seinem Feldbegriff sowie Michel Foucaults Diskurs- und Machtbegriff vollzogen wurde ${ }^{15}$, hatte zum Ziel, die Funktion und

Veröffentlichung eines Mediums (CD, mp3, FLAC usw.) mit klanglich-musikalischem Inhalt sowie die damit einhergehende Flut von Informationen, Anekdoten, Werbeanzeigen, Videos usw., die Interpretationsmöglichkeiten über die Konstruktion von Künstler*innen- oder Band-Images festzulegen suchen. Selbst im Bereich der Hobbymusiker*innen im Internet und ohne Labelanschluss sind Kopplungen von Songveröffentlichungen und weiteren medialen Inhalten relativ üblich.

11 Z.B. Bennet 2009; Barber 2012.

12 Ethnographische Studien basieren auf Daten, die durch teilnehmende Beobachtung erhoben wurden. Die Forschenden bewegen sich dabei gemeinsam mit den Akteur*innen des Feldes in den ausgewählten Bereichen, hier z.B. Tonstudios, und übernehmen teilweise auch bestimmte Rollen, z.B. als Bandmanager*in oder Tontechniker*in. Die mittels Feldnotizen oder Audiomitschnitten festgehaltenen Daten werden dann transkribiert und in Form einer detaillierten Beschreibung veröffentlicht. Idealerweise entstehen so ausschließlich durch die Kenntnis der Daten spezifische Fragen, die dann durch weitere ethnographische Forschungen beantwortet werden können. Zu dieser Form der Forschung hinsichtlich der Tonstudios vgl. Huschner 2016, 120-128.

13 Vgl. z.B. Hennion 1981, 1983 und 1989; Meintjes 2003; Moehn 2005.

14 Es wurde vertraglich vereinbart, dass weder die Studios noch die Akteur*innen im Rahmen meiner Analyse namentlich genannt, sondern nur anonymisiert verwendet werden dürfen, damit ihnen kein beruflicher Nachteil entsteht.

15 Vgl. Bourdieu 1993a; Bourdieu 2005, 49-79; Foucault 2005 und 2013, 74; Huschner 2016, 66-97, 371-379. In der ethnographischen Forschung bezeichnet ,Feld s allgemein den Bereich, in welchem sich das alltägliche Leben der zu untersuchenden Gemeinschaften, Institutionen etc. vollzieht und Daten erhoben werden. Bei Bourdieu ist das >Feldı, vereinfacht dargestellt, ein bestimmter Bereich, in welchem mittels verschiedener Kapitalsorten um die Herrschaft und Deutungshoheit gekämpft wird, wodurch die Strukturen des Feldes ständig reproduziert werden. 
Rolle von Produzent*innen innerhalb der Tonstudios, die klanglichen Bezugspunkte von Verhandlungen der Akteur*innen untereinander sowie deren Auswirkungen zu erklären.

Eine Analyse der während meiner Beobachtungen gehörten Klangkonfigurationen und deren Veränderungsprozesse wäre naheliegend gewesen, konnte aus verschiedenen Gründen aber nicht erschöpfend vorgenommen werden: Einer der wichtigsten Gründe dafür war, dass bereits der wissenschaftlich-ethnographische Zugang zu Studioprozessen ein komplexes und schwieriges Unterfangen darstellte ${ }^{16}$, in dessen Rahmen ein vollständiger Zugriff auf sämtliche Entwicklungsstadien der aufgenommenen Songs aufgrund des Wunsches nach Diskretion der beteiligten Akteur*innen praktisch unmöglich war. Einige diesbezüglich unproblematische Klangfragmente verschiedener Entwicklungsstadien werden jedoch anhand von zwei Beispielen weiter unten vorgestellt. Dementsprechend wird innerhalb dieses Aufsatzes keine exemplarische Analyse einzelner Songs vollzogen oder eine analytische Methodik präsentiert, sondern der Versuch unternommen, den möglichen Mehrwert einer produktionsbezogenen Perspektive bzw. deren Einbeziehung für einen musikanalytischen Zugriff auf populäre Musik allgemein zu verdeutlichen.

\section{ZU EINIGEN SCHWIERIGKEITEN DER ANALYSE VON POPMUSIK}

Um populäre Musik handelt es sich nach Peter Wicke dann, wenn soziale, kulturelle und ästhetische Praktiken »sowohl durch Klang als auch den Warenzusammenhang vermittelt ${ }^{17}{ }^{17}$ werden. Die angesichts dieser Definition in der Vergangenheit geführte Diskussion über die Risiken eines einseitigen musikwissenschaftlich-analytischen Zugriffs ausschließlich auf die klanglichen Erzeugnisse des Popmusikfeldes soll hier nur angedeutet werden: Wickes Aufgreifen einer Kritik Simon Friths bezüglich der Konstruktion eines popmusikalischen `Textes` durch die Verwendung ungeeigneter analytischer Methoden und bezüglich der Fokussierung auf nur sehr begrenzt relevante Aspekte und der damit einhergehenden Erzeugung bestimmter Hörweisen ${ }^{18}$ hat in der Auseinandersetzung mit populärer Musik Widerhall, aber auch Kritik gefunden und zu einer Weiterentwicklung beigetragen. ${ }^{19}$ Wickes Bedenken seien erneut angeführt, weil es in den letzten Jahren einige Versuche gab, bestimmte Qualitäten ausschließlich in der »Musik selbst $\aleph^{20}$ auszumachen, z.B. in Form der Parameter Harmonik, Metrik, Form, mittels Analyse der Funktionsharmonik, Harmoniefolgen, Ursatzlinien nach Schenker usw. ${ }^{21}$ Die zahlreichen

16 Huschner, 2016, 130-136 und passim.

17 Wicke 2004, 166.

18 Wicke 2003.

19 Z.B. Steinbrecher 2016, 42. Der musikanalytische Zugriff wird dabei etwa als nur eine Möglichkeit der Auseinandersetzung mit Popmusik begriffen, vgl. Doehring 2012, 28. Versuche, die Kritik aufzugreifen, finden sich weiterhin bei Doehring 2015, 148-151; Danielsen 2015, 68f.; Zagorski-Thomas 2015, 30; Appen 2015.

20 Zur Kritik am Begriff der >Musik selbst` vgl. Wicke, 2003; Papenburg 2008, 91-94.

21 Vgl. Kramarz 2014; Everett 2015; Riedemann 2012, wobei Riedemann selbst auf die durch die Komplexität »musik- und textimmanenter« Faktoren bedingten Grenzen des Ansatzes verweist (ebd., 53). 
saußermusikalischen Faktoren $^{22}$, die für einen (jeweils zu definierenden) Erfolg in der Popmusikproduktion entscheidend sind, werden bei einer solchen Herangehensweise ignoriert oder marginalisiert. ${ }^{23}$ Als Beispiele seien die Songs Breathe (1996) von Midge Ure und Too Close (2011) von Alex Clare genannt, die beide erst durch die Nutzung als Werbemusik zwei (1998) bzw. ein Jahr (2012) nach ihrer Veröffentlichung und nicht etwa allein durch inhärente `Hitqualitäten berühmt wurden. ${ }^{24}$ Diesbezüglich sei die Produzentin Lauren Christy vom Produktionsteam The Matrix ${ }^{25}$ zitiert:

I have a collection of hit songs that were never hits on my iTunes. To me they were still hit songs but the person got fired at the label or the right indie promotion people weren't put on the job. There are a horrible lot of things that are outside the control of the artist. [...] [This happens] probably $95 \%$ of the time. ${ }^{26}$

Einen rezeptionsästhetischen Ansatz wählt Allan Moore ${ }^{27}$, indem er sich der Frage widmet, wie Hörer*innen bestimmte Songs interpretieren. ${ }^{28}$ Er nutzt dazu die der Psychologie entstammenden Ansätze sembodied cognition` und secological perception ‘ ${ }^{29}$ Grob zusammengefasst lässt sich aus dem Prinzip der secological perception` die Idee ableiten, dass Klangkonfigurationen Informationen bereitstellen, die von Hörer*innen mithilfe von Erfahrungswerten entschlüsselt werden könnten und somit mögliche Interpreta-

22 Huschner 2016, 112f., 199.

23 Neben dieser Problematisierung sei darauf verwiesen, dass Klang nicht zwangsläufig als Träger von Bedeutung wahrgenommen werden muss, um strukturiert zu werden, sondern Hörpraxen existieren, die - vereinfacht formuliert - vor allem auf die sinnliche Erfahrung des Klangs abzielen, weshalb die Redundanz klanglicher Strukturen in der Rock- oder Popmusik für diese Art des Hörens ein Vorteil ist. Eine Art des Hörens, die z.B. von den weiter unten besprochenen Ansätzen Moores und Zagorski-Thomas' nicht adressiert werden kann. Diesbezüglich ist Peter Wickes 14 Jahre alte Kritik noch immer zutreffend.

24 Vgl. Hampp 2012; Midge Ure: http://www.midgeure.co.uk/archive/newsbreathe.html (16.4.2017). Angemerkt sei, dass die Beschaffenheit der Klangkonfigurationen natürlich wichtig ist, um überhaupt das Potential für diese Art der Verbreitung zu haben, eine alleinige Konzentration auf klangliche Faktoren aber der Funktionsweise des Feldes der Popmusikproduktion meiner Meinung nach nicht gerecht wird. Die damit zusammenhängenden Konzepte der `Anschlussfähigkeit und ‘klanglichen Konkurrenzfähigkeit` bezüglich eines potentiellen Funktionierens als Popmusik werden weiter unten thematisiert.

25 Das Produktionsteam besteht aus Lauren Christy, Graham Edwards und Scott Spock und ist in den 2000er Jahren für zahlreiche Erfolge (mit)verantwortlich, so z.B. Avril Lavignes Songs Complicated (2003) und I'm With You (2004); vgl. The Matrix: http://www.thematrixmusic.com/Home.html (27.5.2017).

26 Zit. nach Burgess 2011.

27 Die Auseinandersetzung mit Moore ist von Belang, weil Zagorski-Thomas' The Musicology of Record Production zahlreiche Berührungspunkte mit meinen Forschungen besitzt und sowohl Moores als auch Zagorski-Thomas' Publikation beide mit den eben angeführten psychologischen Konzepten arbeiteten, vgl. Zagorski-Thomas 2014, 27f. Ferner hat Moore das Tonstudio mittels seiner "soundbox« bereits teilweise in die Popmusikanalyse eingebunden, vgl. Dockwray/Moore 2010.

28 Moore 2012, 3.

29 Ich verweise diesbezüglich vor allem auf Moore, da er die Herkunft der Konzepte ausführlich erläutert, vgl. ebd., 12. 
tionsräume begrenzen. ${ }^{30}$ Simon Zagorski-Thomas führt als Beispiel dafür den gewählten Hall einer Produktion an: Besondere klangliche Halleffekte implizierten bestimmte Hallräume. ${ }^{31}$ So können etwa ein besonders großer (eine Kathedrale oder Konzertsaal) oder ein besonders kleiner Raum (ein Wohnzimmer) in Verbindung mit anderen Faktoren die Interpretationsspielräume der Hörer*innen deutlich einschränken. >Embodied cognition` wiederum zielt auf das Anwenden bestimmter bekannter Schemata zur Entschlüsselung von neuen, hier klanglich-kompositorischen Informationen durch Metaphern ab: Zagorski-Thomas verweist z.B. auf die bekannten oder imaginierten Spielpattern auf einer Snare Drum, die gemeinsam mit deren ebenfalls bekanntem Klang (eine Verbindung von secological perception ‘ und ‘cross-domain mapping`) eine bestimmte Interpretation, z.B. ein Militärbegräbnis oder eine Gedenkfeier, nahelegten bzw. anböten. ${ }^{32}$ Diese so eingegrenzten Möglichkeiten der Wahrnehmung von klanglichen Ereignissen in Songs werden von Moore als "affordance « $^{33}$ bezeichnet.

Die Schwierigkeit einer adäquaten Darstellung und die mit der Wiedergabe von Songs in Fünf-Linien-Notation bei Moore ${ }^{34}$ verbundenen Probleme hat Wicke bereits 2003 angesprochen. ${ }^{35}$ Gleiches gilt für das traditionelle Vokabular, mit dem bei Moore bestimmte harmonische Wendungen analysiert werden: »tonic«, »cadence«, »dominant«. ${ }^{36}$ Weiterhin betont Moore zwar mehrfach, dass Songs stets mehrere Aussagen hätten ${ }^{37}$, aber die Wortwahl und eben auch der theoretisch beeindruckende Unterbau seiner Interpretationen verstärken den Eindruck, dass es am Ende doch ein ıbesseres‘, wenn schon nicht alleinig >richtiges Hören geben könnte: Moores Lesart von Bob Dylans All Along the Watchtower (1967) ziele beispielsweise darauf, die bisher ungelösten Probleme mit dem rätselhaften Text zu umschiffen, Tori Amos' Hey Jupiter (1996) sei laut Moore besser als innerer Monolog zu verstehen usw. ${ }^{38}$ Moore beschränkt seine Auseinandersetzung auf Songs mit englischen Texten ${ }^{39}$, wodurch Popmusik ohne Vocals, Hörer*innen mit begrenzten Englischkenntnissen und Songs, die in Fantasiesprache gesungen werden (z.B. Hjómalind von Sigur Rós, 2007), inhaltlich kryptisch sind (z.B. Black Hole Sun von

30 Ebd., 243-248.

31 Zagorski-Thomas 2014, 47f.

32 Ebd., 9-13.

33 Moore 2012, 6, 12 und passim.

34 Ebd., z.B. 116, 234, 240.

35 Wicke 2003.

36 Moore 2012, 226, 233, 235, 296 und passim.

37 Ebd., 12, 330 und passim.

38 Ebd., $240 f ., 252$.

39 Ebd., 3. 
Soundgarden, 1994) oder deren Texte vor allem eine rhythmische und nicht inhaltliche Funktion erfüllen ${ }^{40}$, von seinen Erklärungen ausgeschlossen werden. ${ }^{41}$

Zagorski-Thomas widmet sich umfassend den diversen Einflüssen von Tonstudios auf die Rezeption von Popmusik, z.B. in Form von »sonic cartoons«. Damit sind Übertreibungen von bestimmten Frequenzen oder Kombinationen von Instrumenten, Hallräumen oder spezifischen Klängen gemeint, die Rezipient*innen inzwischen weitgehend fraglos akzeptieren. ${ }^{42}$ Obwohl ich Zagorski-Thomas' Kritik an der mangelnden Angemessenheit vieler verfügbarer analytischer Instrumente der Popmusikanalyse teile ${ }^{43}$, scheint mir seine nicht-modifizierte Übernahme bzw. Kombination zahlreicher verschiedener Ansätze wie u.a. der Akteur-Netzwerk-Theorie (ANT), der ssocial construction of technology (SCOT) oder dem ssystems approach to creativity $\iota^{44}$ problematisch zu sein. Die Nutzung von Begriffen Pierre Bourdieus, z.B. die verschiedenen Kapitalsorten, samt damit verbundenem bzw. impliziertem theoretischen Überbau ${ }^{45}$, in Verbindung mit der Akteur-NetzwerkTheorie Bruno Latours ist angesichts der Kritik Latours an der kritischen Soziologie und der ANT als Gegenentwurf zu dieser zumindest diskutabel. ${ }^{46}$ Trotzdem spricht ZagorskiThomas wichtige Aspekte an: Etwa die Notwendigkeit der Kenntnisse der für die Analyse notwendigen soziokulturellen Kontexte auf Produktions- sowie Rezeptionsseite, die Frage nach den Maßstäben von Bewertungen innerhalb der Produktionsprozesse oder die Forderung nach ethnographischen Studien über Hörer*innen und Produzent*innen. ${ }^{47}$

Man kann also zwei gängige Ziele der Analyse von Popmusik erkennen: Erstens die Suche nach den inhärenten Hitqualitäten, also die klanglich-kompositorischen Faktoren für angestrebten ökonomischen Profit - ein Aspekt, den ich aus den genannten Gründen hier nicht weiter verfolgen werde. Zweitens die Versuche, plausible Interpretationen ausgewählter Songs anzubieten, wie es z.B. Moore und indirekt Zagorski-Thomas vorschlagen. Ich möchte noch ein drittes Ziel formulieren: Die Analyse von Popsongs in Verbindung mit Analysen des soziokulturellen Entstehungskontextes, um die Funktionsweise

40 Ina Plodroch thematisiert diese bereits länger stattfindende Entwicklung: „Und worüber die Band da singt - wen interessiert das schon? « (Plodroch 2017) und zitiert eine Quelle hinsichtlich der Bedeutung der Texte folgendermaßen: „Das beste Beispiel ist wohl [Max Martins] erster Nummer-EinsSong 'Hit me Baby (One More Time) « von Britney Spears. Die Zeile ist einfach Quatsch, es müsste heißen: hit me up one more time. Als der Song rauskam, wusste niemand, was das heißen soll. Aber das war egal. Es machte ihn als Hit sogar noch attraktiver.« (ebd.)

41 Bei aller Kritik sei angemerkt, dass ich das Unterfangen der Popmusikanalyse als dezidiert musikwissenschaftliche Aufgabe ansehe und somit die hier kritisierten Ansätze trotzdem als sinnvoll erachte, um der Lösung der genannten Probleme näher zu kommen.

42 Zagorski-Thomas 2014, 49-69.

43 Ebd., 156.

44 Ebd., 4. Dass es sich eher um eine Sammlung verschiedener Zugangsmöglichkeiten und nicht um ein wirkliches Modell handelt, thematisiert auch Elsdon $(2016,178)$.

45 Weiterhin der Feld- oder Habitus-Begriff, vgl. Zagorski-Thomas 2014, 88, 116, 130, $209-211$.

46 Selbstverständlich kann die ANT zur Nachzeichnung von Studioprozessen genutzt werden, meine Kritik richtet sich hier auf Zagorski-Thomas' Kombination mit Elementen der kritischen Soziologie, gegen die Latour entschieden argumentiert, vgl. Latour 2010, 7, 146, 269.

47 Zagorski-Thomas 2014, 22f., 36f., 64f., 77. 
des Feldes der Popmusikproduktion und seiner diesbezüglichen Veränderungen in den beobachteten Bereichen darstellen zu können.

Die zwei letztgenannten Ziele profitieren vom Hinzuziehen der Produktionsperspektive: Moore thematisiert den Einfluss der Studioakteur*innen ${ }^{48}$ und merkt an, dass u.a. auch die Stabilität der Beziehungen zwischen Produzent*innen, Tontechniker*innen und Musiker*innen zu berücksichtigen sei. Dies wäre mangels detaillierter Kenntnisse der Prozesse aber nur selten möglich. ${ }^{49}$ Gleichzeitig ist das Tonstudio ein Ort, an dem der (ideologische) Einfluss des Popmusikfeldes in Form von Diskussionen über Klänge, deren Bewertung und Deutung in Bezug auf andere Klangkonfigurationen sowie die hauptsächlich mit der eigenen Musik verbundenen Hoffnungen auf ökonomischen Erfolg deutlich sichtbar werden ${ }^{50}$, weswegen Moores Anmerkung, "the market" sei für sein Anliegen irrelevant ${ }^{51}$, überrascht.

Die klanglichen Resultate entstehen im Zusammenwirken und im Zuge von Verhandlungen zwischen Musiker*innen oder Bands und Studioakteur*innen. Während es in der `Electronic Dance Music aufgrund der nahezu ausschließlich elektronischen Musikentstehungsprozesse ggf. angemessen erscheint, die Publikationen den veröffentlichenden Künstler*innen zuzuschreiben ${ }^{52}$, ist die durch Frith angesprochene Idee der Kommunikation der verschiedenen Konstellationen von Künstler*innen oder Bands und Produzent*innen mit den Hörenden problematisch, weil deren Anteil am Ergebnis unklar ist. ${ }^{53}$ Zudem ist Moore zuzustimmen, dass es schwerlich nur eine `Botschaftı geben kann, die kommuniziert wird ${ }^{54}$, vielmehr dürften die jeweiligen Songs oder Tracks gemeinsam mit außermusikalischen Faktoren (Interviews, Videos usw.) Teil eines zu kommunizierenden Images sein, welches wiederum die Interpretation der Hörenden beeinflusst. ${ }^{55}$ Dass es in der nichtakademischen Rezeption nach wie vor das Bild von sechten Musiker*innen bzw. Künstler*innen gibt, die ihre Songs und Texte ausschließlich selbst schreiben und daher sauthentisch sind ${ }^{56}$, zeigt eine der jüngsten Fernsehsendungen des Moderators Jan Böhmermann, in der er in satirischer Zuspitzung den Aussagen des Sängers Max Giesinger zu seinem Songwriting eine Auflistung der zahlreichen beteiligten Akteur*innen

48 Ich unterscheide zwischen Studioakteur*innen, wie z.B. Tontechniker*innen und Produzent*innen, deren Lebensunterhalt hauptsächlich durch diese Tätigkeiten bestritten wird und die dementsprechend über ein hohes Maß an spezifischem Wissen verfügen, und Bands oder Musiker*innen, die nur für relativ kurze Zeitabschnitte im Studio agieren, obwohl diese für diesen Zeitraum natürlich auch als 'Studioakteur*innen، bezeichnet werden könnten.

49 Moore 2012, 180, $212 \mathrm{f}$.

50 Huschner 2016, 371-379.

51 Moore 2012, 214.

52 Doehring 2015.

53 Die Unterscheidung zwischen Künstler*in, Kunstfigur, Produzententeam oder Fragen nach den verschiedenen Formen der Authentizität seien hier nur am Rande erwähnt.

54 Moore 2012, 12.

55 Ebd., 179-214, 259-271.

56 Moore hat sich bereits vor Song Means ausgiebig mit diesem Konzept auseinandergesetzt (vgl. Moore 2002), ich verzichte hier auf eine eingehendere Auseinandersetzung mit diesem. 
gegenüberstellt. ${ }^{57}$ Dies verdeutlicht vor allem die ideologische Diskrepanz zwischen projiziertem Wunschbild von Hörerklientelen sowie evoziertem Bild von Plattenfirmen und den tatsächlichen Arbeitsweisen innerhalb des Feldes der Popmusikproduktion. So agiere laut Ina Plodroch ein Indie-Musiker unter Pseudonym, wenn er Schlager-Hits oder Dance-Pop für andere Künstler*innen schreibe, um seine Glaubwürdigkeit als Musiker zu bewahren. ${ }^{58}$

Obwohl man sich bewusst nicht mehr nur jenen Songs oder Genres widmet ${ }^{59}$, die sich besonders für bestimmte analytische Zugriffsformen eignen oder einen allgemein postulierten, besonders hohen Wert haben ${ }^{60}$, besteht weiterhin die (nachvollziehbare) Tendenz, sich primär auf öffentlich wahrgenommene bzw. erfolgreiche Bands, Musiker*innen oder Songs zu konzentrieren. ${ }^{61}$ Ein Fall, in dem diese Aspekte zusammenkommen und partiell auch thematisiert werden, ist Ralf von Appens fiktive Entstehungsgeschichte von Ke\$has Tik Tok (2009). Er verweist auf die Relevanz der Darstellungsintention der an der Entstehung dieses Songs Beteiligten, die deutlich voneinander - auch in der Bewertung des Ergebnisses - differieren könnten und deren rückblickenden Aussagen zudem nicht immer zuverlässig seien. ${ }^{62}$ Zagorski-Thomas benennt ebenfalls Überlieferungsprobleme, bei denen man mangels Alternativen den Erzähler*innen bzw. deren Darstellung des Geschehens Glauben schenken müsse. ${ }^{63}$

Eben diese für die finale Form der Klangkonfigurationen relevanten Prozesse in Tonstudios, die durch die Spezifik der Konstellationen der Akteur*innen bedingt sind, werden nur höchst selten dokumentiert. Will man Erzeugnisse der Popmusik auch aus der Produktionsperspektive untersuchen, ist man fast immer von retrospektiven Darstellungen abhängig, welche die Vorgänge und Konflikte in den Studios nicht unverfälscht darstellen können, wollen oder sollen. ${ }^{64}$ Da solche Aktivitäten meist marketingbezogenen bzw. profitorientierten und nicht wissenschaftlichen Interessen entspringen, leuchtet das auch ein. Angesichts des hohen Ausstoßes an Publikationen im Popmusik-Bereich ${ }^{65}$ und dem nur geringen Prozentsatz davon, der dann tatsächlich profitabel wird, zeigte sich indes Simon Frith schon vor Jahren über die Schwerpunktsetzung auf vor allem erfolgreiche Publikationen verwundert: »[R]ecord companies spend a considerable amount of

57 Böhmermann 2017, ab 2:49.

58 Plodroch 2017.

59 Appen/Doehring/Helms/Moore 2015, 2.

60 Z.B. die Beatles oder Progressive Rock im Allgemeinen. Vgl. dazu u.a. Everett 1999 und 2001; Spicer 2000; Keister/Smith 2008, 448, 450.

61 Vermutlich hängt dies mit dem (berechtigten) Anspruch der Musikwissenschaft zusammen, klangliche Phänomene, die finanziell erfolgreich sind, zumindest teilweise erklären oder nachzeichnen zu wollen.

62 Appen 2015, 46.

63 Zagorski-Thomas 2014, 104, 155, 178, 186.

64 Vgl. z.B. Morrow 2012.

65 Bundesverband Musikindustrie 2017, 19. 
time producing sfailuress, and yet there are very few studies of these failures - the spublic verdict is allowed a retrospective authority. ${ }^{66}$

Um vorher herauszufinden, welche Diskurse und Codes das Klanggeschehen strukturieren und aus welchem Grunde ${ }^{67}$, wäre, wie auch André Doehring feststellt ${ }^{68}$, eine ethnographische Datenerhebung sinnvoll, damit Analysen der Produktion nicht nur auf handwerkliche Aspekte begrenzt bleiben. ${ }^{69}$ Zudem würde dem Problem der fehlenden schriftlichen Fixierung von Popmusik und dem dadurch bedingten Mangel an Nachvollziehbarkeit der Entstehungsprozesse immerhin teilweise entgangen werden. Eine gewisse Loslösung vom Zwang der Auseinandersetzung mit bekannten Songs bzw. Bands oder Produzent*innen ist, wenn man auf plausible Interpretationen von Songs und die Funktionsweise des Feldes abzielt, unproblematisch. Das erschwert zwar deutlich die Materialbeschaffung, für den Zugang zu diesem ohnehin schwierigen Feld der Klangkonfigurationserzeugung ${ }^{70}$ ist die Konzentration auf noch nicht (besonders) erfolgreiche Tonstudios bzw. Bands oder Musiker*innen aber im doppelten Sinne sinnvoll: (1) Man hat leichteren Zugang zu entsprechenden Einrichtungen bzw. Sessions. Natürlich ist man als Feldforscher*in vor allem auf diejenigen Studios und Bands oder Musiker*innen angewiesen, die zur Kooperation bereit sind, und vermutlich sinkt deren Bereitschaft dazu mit zunehmendem Erfolg. Allerdings dürfte es angesichts der relativ hohen Zahl von Musikwissenschaftler*innen, die in verschiedenen Positionen in der Popmusikproduktion tätig sind, nur eine Frage der Zeit sein, bis entsprechendes Material auf breiterer Basis vorhanden ist. Zudem ist es auch mir als Außenstehendem gelungen, über erweiterte persönliche und berufliche Beziehungsnetzwerke in renommierten Berliner Tonstudios Einblick in die Produktionsgepflogenheiten verschiedener Projekte zu erhalten, sodass der Schwerpunkt meiner Forschung darauf lag, Beobachter bei den Entstehungsprozessen eines noch nicht erschienenen Albums oder Songs zu sein. Daneben ist (2) die Frage nach einer zukünftigen (massenhaften) Rezeption und dem damit einhergehenden (finanziellen) Erfolg grundsätzlich offen und soll zumindest für die beteiligten Akteur*innen im Tonstudio - bezogen auf die Konfiguration der Klänge und antizipierten Feldprozesse - so präzis und zugleich so offen wie möglich beantwortet werden.

\section{DIE PRODUKTIONSPERSPEKTIVE: ANSCHLUSSFÄHIGKEIT, ARBEITSTEILUNG, SELBSTVERORTUNG UND SOUND}

Im Folgenden gehe ich auf einige Interaktionen oder Wortäußerungen in den von mir beobachteten Sessions ein, die mir bezüglich der formulierten Erkenntnisinteressen gewinnbringend erscheinen. Die angeführten Beispiele berühren zwar stets mehrere

66 Frith 1998, 60.

67 Wicke 2003.

68 Bei Doehring $(2012,29)$ ist vermutlich die ethnographische Datenerhebung im Bereich der Konsumierenden gemeint.

$69 \mathrm{Vgl}$. die lesenswerten Auseinandersetzungen mit Songs und ihren produktionsbezogenen Besonderheiten z.B. bei Zagorski-Thomas 2015 oder Appen 2015.

70 Huschner 2016, 120-128. 
Ebenen der Studioprozesse bzw. gehen teilweise von einer Ebene in eine andere über, sollen hier aber im Sinne der Nachvollziehbarkeit einzelnen Aspekten zugeordnet werden. Zunächst werden die Begriffe `Anschlussfähigkeit` und `klangliche Konkurrenzfähigkeit erläutert, da sie für die Studioprozesse prägend sind. Anschließend gehe ich auf das Problem der unklaren Arbeitsverteilung innerhalb der Studioprozesse ein, da die Mitwirkung am Entstehungsprozess häufig notwendiger Teil des Künstler*innen-Images ist und somit auch die Rezeption von Klangkonfigurationen betrifft. Die anschließend von mir thematisierte Selbstverortung der Künstler*innen hat direkten Einfluss auf diese Verteilung der Befugnisse und damit auf Prozesse der Klangerzeugung in den Tonstudios. Anschließend folgen Überlegungen zur Datenerhebung bezüglich der Entwicklung und Veränderung der in den Studios erzeugten, veränderten und konfigurierten Klänge. Abschließend stelle ich konkrete Klangbeispiele vor, an denen die Veränderungen des Sounds von zwei Songs einer beobachteten Band nachvollzogen werden können, um die Auswirkungen der zu Beginn geklärten Begriffe `Anschlussfähigkeit‘ sowie `Konkurrenzfähigkeit` zu verdeutlichen.

\section{sAnschlussfähigkeitı und sklangliche Konkurrenzfähigkeit‘}

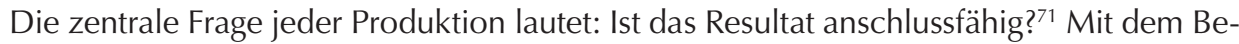
griff `Anschlussfähigkeit` ist die meist massenhafte Nutzung der Erzeugnisse in bestimmten, antizipierten Kontexten gemeint, um kommerziell erfolgreich bzw. rentabel zu sein. Eine Möglichkeit ist die Unterbringung von Tracks bei Fernsehserien ${ }^{72}$, die Konzeption als Tanzmusik, als Stadionhymne ${ }^{73}$, als Mittel zur (musikalisch-klanglichen) Abgrenzung von anderen Tracks, als Klangevent in Form von Konzerten und anderen Hörereignissen oder immer häufiger die Verwendung in Computerspielen ${ }^{74}$ usw. Die Frage nach der Anschlussfähigkeit stellt sich nicht nur in Form von kompositorischen, sondern vor allem von klanglichen Eigenschaften. Der Schlagzeuger einer von mir beobachteten Band dazu:

Den Versuch, [uns im Radio] zu platzieren, hatten wir mit einem Song gestartet, [...] der hatte alles [geläufige Songform, einprägsame Hook ${ }^{75}$, professionelle Produktion,

71 Die Begriffe `Anschlussfähigkeit‘ und 'klangliche Konkurrenzfähigkeit stammen von mir und fassen die implizierten, beobachteten und formulierten Ziele der verschiedenen Studioakteur*innen und Musiker*innen innerhalb verschiedener Produktionen zusammen.

72 Z.B. der Song Hey Now (2013) von London Grammar oder Intro (2009) von The XX in der Serie Suits (Tunefind, http://www.tunefind.com/show/suits/season-3/15361 (28.02.2017).

73 Huschner 2016, Anhang 12.

74 Z.B. Lacuna Coil mit Swamped (2004) in Vampire: The Masquerade - Bloodlines (2004), das Shawn Lee's Ping Pong Orchestra mit Kiss The Sky (2006) in Tales from the Borderlands (2014-2015) oder die FIFA-Fußballspielserie von Electronic Arts mit diversen Titeln (meines Wissens seit FIFA 97, 1996). Während es sich dabei stets um Lizensierungen handelte, gibt es auch andere Varianten. So hat die Band 65daysofstatic für das Spiel No Man's Sky den Soundtrack No Man's Sky: Music For An Infinite Universe (2016) beigesteuert.

75 Mit ’Hook ist ein Teil eines Songs gemeint, welcher Hörer*innen zum Weiterhören animieren soll, also frei übersetzt »an den Haken nimmt» und nicht mehr loslässt. Hooks können beispielsweise 
Musikvideo], was man fürs Radio gebraucht hätte und wurde extra dafür so geschrieben. Wir wissen nicht, warum es nicht geklappt hat. ${ }^{76}$

Ein Produzent zum Problem des >richtigen Sounds einer Bass Drum:

Das ist gar nicht so einfach, ich hab' da tierisch lange dran rumgebastelt an diesem einen [Sound]. [...] Das klingt total simpel, also das klingt so, als [...] könnte man [das] in 'ner halben Stunde machen ${ }^{77}$, ich hab' da bestimmt dreißig Stunden dran gesessen, damit das - tausend Bass-Drums gehabt, Bässe, Bass-Sounds, weil das muss alles zusammenpassen halt $[\ldots]$.

Derselbe Produzent zur Anschlussfähigkeit eines Fan-Liedes:

Also ich hab' so 'ne Version gemacht, die klingt halt sehr elektronisch. Und dann der, äh, Stadion-DJ meint: »la ok, ist super! « Aber er würde lieber, also er selber steht mehr auf Rock und er würde auch sagen, viele Fans würden auch auf Rock stehen halt. Obwohl die Fans, die haben den Song schon sozusagen angenommen, die fanden den auch schon total gut, also der Fanclub. [...] jetzt... versuch' ich, den trotzdem bisschen rockiger zu gestalten, halt, ja?

Anschlussfähigkeit bedeutet zugleich auch klangliche Konkurrenzfähigkeit, die in diversen Formen in Erscheinung treten kann, so etwa mittels bewusster Verringerung der Klangqualität ${ }^{78}$, des spezifischen Sounds eines Songs insgesamt ${ }^{79}$ oder der vermittelten Echtheit des Höreindrucks, z.B. durch wahrnehmbare Atmung der Singenden. ${ }^{80}$ Die jeweiligen klanglichen Bezugspunkte, also die präferierten Songs, Bands oder Musiker*innen, und die Weisungsbefugnisse der Vertreter*innen dieser Bezugspunkte entscheiden, in welche Richtung die Klangkonfigurationen bearbeitet werden, und es kann vorkommen, dass Rocksongs mithilfe von Songs von Britney Spears oder Depeche Mode konkurrenzfähig gemacht werden ${ }^{81}$ oder komplette Gitarren noch einmal aufgenommen werden müssen, weil sie für den/die Produzent*in in bestimmten Spielweisen zu sehr nach `Classic Rockı klingen würden. ${ }^{82}$

instrumentale oder vokale Linien, auffälliges Sound-Design, ein Gitarren-Riff oder ein SchlagzeugPattern sein. Vgl. Burns 1987 zur Funktion unterschiedlicher Elemente in verschiedenen Songs als 'Hooks.

76 Alle Zitate entstammen transkribierten Gesprächen während beobachteter Sessions und wurden nur geringfügig für die Lesbarkeit (z.B. Stotterer, Versprecher usw.) korrigiert. Starker Dialekt oder Umgangssprache fielen nicht in diesen Bereich.

77 Der Produzent verweist hier auf den vermeintlich schnell gefundenen Sound durch Auswahl eines Samples aus vorgefertigten Sample-Bibliotheken und ein relativ einfaches Pattern des programmierten Schlagzeugs. Sein erklärtes Ziel war es, mit so wenig Elementen wie möglich ein befriedigendes Hörerlebnis zu schaffen.

78 Zagorski-Thomas 2010, 259f.; Hodgson 2010, 90, 294.

79 Huschner 2016, 159f., 170, Anhang 21.

80 Weinstein 2016, 120.

81 Huschner 2016, 352, Anhang 152.

82 Ebd., Anhang 136. 
Die klangliche Konkurrenzfähigkeit wurde bei den Sessions einer Jazzcombo hergestellt, indem beispielsweise in bestimmten Passagen das Klangbild mittels Equalizern $(\mathrm{EQ})^{83}$ oder Stummschaltung bestimmter Instrumente bereinigt wurde, z.B. nur auf den tiefen Passagen der Main-Vocals für deren Verstärkung ein Kompressor genutzt wurde, Übersprechungen ${ }^{84}$ durch Edits beseitigt wurden oder indem der Produzent den Schlagzeugsound einer gründlichen Überarbeitung unterzog. ${ }^{85}$ Dass die Vorstellungen bezüglich dieser Konzepte je nach Zielstellung und Selbstverortung der Beteiligten und der verfügbaren Ressourcen deutlich auseinandergehen können, sollte klar sein.

\section{Arbeitsteiligkeit und ihr Einfluss auf das Resultat}

Die Verteilung der Prozesse in der kollaborativen Erarbeitung von Klangkonfigurationen variierte in den beobachteten Sessions stark: In einer Session hatte der Sänger einer Formation ausdrücklich den Wunsch geäußert, bei der Produktion ${ }^{86}$ seiner neuesten Song-Idee dabei zu sein. Letztendlich saß der Sänger dann leicht angetrunken im Studio, döste hauptsächlich und gab in unregelmäßigen Abständen kurz seine Zustimmung zum klanglichen Geschehen, während der Produzent kompositorische, klangliche und technische Aspekte bearbeitete (Basslinien, Drum-Grooves, C-Teil; im Prinzip komponierte und arrangierte der Produzent die Songs im Alleingang).

In Studiosituationen mit der bereits erwähnten Jazzcombo wiederum folgte deren Produzent, der nicht als solcher von den Bandvertreter*innen bezeichnet wurde, in den meisten Fällen präzise den Vorgaben der Musiker*innen bei allen Bearbeitungen und griff nur minimal in die kompositorischen Geschehnisse ein, bearbeitete dafür indes vor allem die klanglich-technischen Parameter zwecks ihrer Konkurrenzfähigkeit, wie oben erläutert wurde.

Bei einer Gitarrensession in einem Projektstudio schließlich wirkte der eigentlich als Tontechniker agierende Studioinhaber (T.) aufgrund seiner Erfahrungen als studierter Gitarrist auf den Einspielenden (G.) immer energischer ein und forderte schließlich explizit bestimmte Spielweisen und Ideen ein, da der Gitarrist mit einer erst im Studio entwickelten längeren Form des einzuspielenden Songs überfordert war. Dies mündete vor den nächsten Einspielversuchen in folgendem Dialog:

T: (recht laut und energisch) Spiel' einfach $\mathrm{n}$ paar einzelne Töne, spiel' doch einfach mal so ne ganz, ganz leichte Melodie! Und dann lassen wir die laufen, dann spielst Du

83 Equalizer sind Geräte oder Software, die verschiedene Filter in sich vereinen, mit denen das Eingangssignal entsprechend bearbeitet werden kann. So können z.B. die Frequenzen ab ein bis zwei $\mathrm{kHz}$ eines einzelnen Signals oder gesamten Mixes punktuell oder allgemein angehoben werden, um einen brillanteren Klangeindruck zu erzeugen.

84 Wenn mehrere Instrumente (z.B. Schlagzeug, Bass, Bläser) in einem Raum aufgenommen werden und auf den Spuren der Bläser das Schlagzeug zu hören ist und vice versa, spricht man von »Übersprechungen «.

85 Huschner 2016, Anhang 70-80, 102-119.

86 Zur Bedeutung des Begriffs »Produktion« vgl. u.a. Howlett 2012; Burgess 2005; Huschner 2016, 97-110, 356-371. 
darüber wieder was, dann spielste darüber wieder was und [...] ich [werde das] so 'n bisschen pannen und mach' das groß. [...] jetzt haste noch nie U2 gehört? [...]

G: (guckt T. irritiert und genervt an) Ja klar, T., wat soll denn dit?! Wat is'n dit für ne Frage überhaupt?

T: Na davon red' ick doch seit ner-

G: Ick bin doch kein Schüler!

T: Ja - Hä? Wat soll denn dit jetzt? Dann weeßte doch, wovon ick rede! [...]

G: Ja, ick hab so was aber noch nie gemacht! [...]

In allen drei Fällen erschienen die Resultate unter dem jeweiligen Band- bzw. Künstler*innennamen, obwohl die Anteile am Entstehungsprozess sich jeweils unterschiedlich darstellten. Diese Unterschiede hätten z.B. hinsichtlich möglicher Interpretationsspielräume oder der Rezeption deutliche Auswirkungen, denn der Sänger des ersten Beispiels wird für sein natürliches Auftreten und seine charmanten Texte gelobt. ${ }^{87}$ Bei Konzerten, von denen ich eines besuchte, brachen Fans bei eher traurigen Songs durchaus in Tränen aus. Weiterhin wird vom Sänger viel Wert auf eine Wahrnehmung gelegt, die sich mit Moores «first person authenticity « ${ }^{88}$ am ehesten beschreiben lässt. Diese Konstruktion - so ist auf seiner Webpräsenz u.a. zu lesen, dass ausschließlich Eigenkompositionen gespielt werden würden - kollidiert aber mit seiner Abhängigkeit von den technischen und instrumentalen Fähigkeiten seines Produzenten, dessen Name nirgendwo auftaucht. Diese extreme Abhängigkeit wiederum berührt die Befugnisse innerhalb der Tonstudios, die letztendlich für die Erscheinungsformen der Songs verantwortlich zeichnen, welche wiederum als klanglich-stilistische Bezugspunkte Auswirkungen auf andere Produktionen vergleichbarer Formationen haben können usw.

Die zweite Session verdeutlicht, wie Positionierungen im Tonstudio diskursiv konstituiert werden, die hier zweifach auf die Erzeugnisse der Sessions wirkten: Zunächst ist das Selbstbewusstsein von studierten Musiker*innen mit akademischen Titeln, die im Jazz-Bereich agieren, meinen Beobachtungen zufolge deutlich höher, was auch an ihren instrumentalen Kompetenzen (etwa im Vergleich zu den Kompetenzen des Sängers im ersten Beispiel) liegen dürfte. Eingriffe in kompositorische Aspekte standen aufgrund des Selbstverständnisses der Band nie zur Debatte, während maßgebliche klanglich-technische Veränderungen außerhalb der eigenen Expertise als weniger wichtig hingenommen wurden; das Verhältnis zwischen Musiker*innen und Produzent*in stellte sich also völlig anders dar. Hier wäre z.B. interessant, ob der von den Musiker*innen gesetzte Schwerpunkt ihrer Selbstverortung auf der Kompositionsebene den Interpretationsrahmen der Rezipient*innen mitbestimmt und inwieweit klangliche Vorstellungen und Bezugspunkte, etwa der Wunsch nach einer weitgehend ohne Effekt erklingenden Gitarre, die Rezeption prägen.

Der dritte Fall schließlich zeugt vor allem von einer noch nicht etablierten Hierarchie zwischen Tontechniker und Musiker ${ }^{89}$, wodurch sich ein offener Konflikt ankündigt.

87 Huschner 2016, Anhang $10 f$.

88 Moore 2012, 269.

89 Huschner 2016, 94-115. 
Zudem ist zu fragen, ob eine zu deutliche Orientierung an U2, einem offenbar positiv konnotierten Bezugspunkt für Gitarrenspielweisen mit wenig melodischer Bewegung, aber viel Effektanteil, der hier gefordert wurde, dem Endprodukt nicht zum Nachteil gereichen würde. Untersuchenswert wäre etwa die klangliche Struktur anhand derart expliziter Bezugspunkte, weil sich dann die Frage stellt, ob die Grenze zwischen Plagiat, Inspiration und Hommage überschritten wurde und ob das für die Anschlussfähigkeit des Songs und für die Rezeption problematisch ist. Ferner werden an diesem Beispiel auch Diskrepanzen im Umgang mit Feldprozessen deutlich: Während der Tontechniker eine klare Vorstellung davon zu haben scheint, was für ein klangliches Material er benötigt, um den Song mithilfe des Tonstudios zum Abschluss zu bringen, fehlt dem Gitarristen diese Vision offenbar - ein Grund, warum der Tontechniker seine Vorstellungen letztendlich durchsetzen konnte und dadurch einen größeren Anteil am Ergebnis hatte als der Gitarrist der Band..$^{90}$

\section{Selbstverortungen}

Der Wille zur Abgrenzung vom wahrgenommenen Mainstream ist nicht spezifisch für Musiker*innen des Jazz-Feldes. Es finden sich auch bei Pop- oder Rockbands immer wieder Situationen, in denen sowohl Musiker*innen als auch Studiopersonal mit kompositorischen Ideen, Formen, Stimmführungen etc. für einen vermeintlichen Sonderstatus argumentieren. So formulierte der Schlagzeuger S. einer Pop-Band mir gegenüber:

Das ist jetzt das zweite Album, wenn es jetzt nicht spürbar nach vorne geht - damit meine ich nicht den großen Durchbruch, aber man sollte schon merken, dass was passiert - dann muss man sich um die Zukunft des Projekts Gedanken machen. Vor allem, weil das Album kompositorisch nochmal viel besser [als das erste Album] ist!

Tatsächlich wiesen die Klangkonfigurationen einige unübliche Besonderheiten auf: Ein Song begann in einem sehr langsamen Tempo und zog dann über längere Zeit passend zur dramatischen Steigerung des Textes immer mehr an. Ein anderer Song begann im Tempo 176 bpm und wechselte etwa bei der Hälfte in ein deutlich langsameres Tempo (127 bpm), wobei der Groove sich völlig veränderte, die gesungene Melodie aber auf beiden Songhälften gleichblieb. Ungerade Taktarten kamen in einem Großteil der Songs vor und die Sprünge zwischen Stilen innerhalb von Songs wurden vom Schlagzeuger als Qualitätsmerkmal angesehen (»[Das ist ein] absolut cooler Song. Mit bisschen Disco, bisschen Jazz, bisschen Metal, Hard-Rock, ja...«).

Die Bezeichnung >Pop-Band wurde von der Band selbst nicht akzeptiert, sie verortete sich im Bereich des Indie-Pops und suchte sich u.a. mittels der genannten ungewöhnlichen musikalischen Wendungen von anderen Bands und Songs abzusetzen. ${ }^{91}$

90 Die Session wurde nicht veröffentlicht. Es handelt sich um die Session vom 24.3.2013, Huschner 2016, 403.

91 Huschner 2016, Anhang 62. 
Solche (Selbst-)Zuordnungen sind stets problematisch diffus ${ }^{92}$, zeugen aber vom grundsätzlichen Prinzip der Absetzungsbestrebungen der Kulturproduzent*innen voneinander, das Bourdieu beschreibt. ${ }^{93}$ Im Tonstudio wird ersichtlich, mit welcher Absicht und mit welchen Vorstellungen, die von den bisherigen Erzeugnissen des gewählten (Teil-)Feldes innerhalb der populären Musikproduktion strukturiert werden ${ }^{94}$, Künstler*innen und Produzent*innen bestimmte klangliche Ereignisse, deren technische Erzeugung sowie Bearbeitung und kompositorische Ideen als passend ein- oder ausschließen. Es wäre in diesem Fall durchaus möglich, die angeführten Songbeispiele harmonisch-metrisch-melodisch zu untersuchen, um z.B. die neue Strukturierung der gleichbleibenden Melodie in zwei verschiedenen Tempi und den intendierten Reiz für Hörende herauszuarbeiten. Allerdings wäre eine solche Herangehensweise verkürzt: Der maßgebliche Einfluss von Technik auf die Prozesse der Klanggenerierung ${ }^{95}$ würde ebenso unberücksichtigt bleiben wie die Frage nach dem srichtigen s Sound und seiner Lesart sowohl auf Produktionsals auch auf Rezeptionsseite. Die größten Konflikte während der hier genannten CDProduktion entstanden nicht etwa wegen jener kompositorischen Besonderheiten, die mit zu antizipierenden Hörgewohnheiten brachen, sondern dann, wenn der Sound der Drumrecordings des Studios des Bandproduzenten mit dem Sound der von mir beobachteten Session in einem anderen Studio verglichen bzw. der Sound oder die Bearbeitung generell thematisiert wurde $(S=$ Sören, Schlagzeuger; $F=$ Felix, Tontechniker):

S: [Die alte Aufnahme] Ist 'ne Katastrophe, man! Ich hab' das [im Studio des Produzenten] nicht gehört! [...] [Die neu aufgenommene] Snare, muss ich sagen, Felix, die bleibt druff. Find' ich richtig gut!

F: Okay, ich mag [den Sound]! Ich mag [den] echt gern!

S: Die macht einen dermaßenen Alarm da! Herrlich! [...] Ja, scheiße man! [...] Also, wirklich, das ist alles in Ordnung beim [Produzenten der Band], aber das mit dem Drum-Set aufnehmen macht mich fertig. [...] Letztlich gesehen, das Schlimmste an den ganzen Recordings bin ja sowieso ich. Ja? Das ist einfach [...] was [den Produzenten] fertigmacht. Dass ich ins Studio komme und diese ganze Kabellage und so, da verliert er die Nerven. [...]

F: Ich hab' ne ganz abgespacete Idee. Ich hab' jetzt zwei (Drum-Takes) aufgenommen, wenn du die echt tight spielst, ja, und kann das jetzt exakt aufeinander schneiden, könnte man im Mix die Idee haben, zweimal Mono-Drum-Tracks zu nehmen: Einen komplett links, einen komplett rechts. [...]

S: Also, äh, zwei völlig verschiedene Drum-Sets, eins ganz scharf links, eins ganz scharf rechts? [...] Hab' so was noch nie gehört. [...] Da haste rechts mal anders als links.

F: Ist doch scheißegal!

S: Ey, Felix, das stört mich. Ist komisch.

92 Wicke 2004.

93 Bourdieu 1993b, 148, 152.

94 Bourdieu 2001, 371-378.

95 Zur Relevanz technischer Aspekte bei der Analyse von Popmusik vgl. z.B. Papenburg 2008, 103 106 und bezogen auf den Einfluss auf das Klanggeschehen Zagorski-Thomas 2014. 
F: (atmet hörbar ein) Ich würd's mal im Hinterkopf behalten, [...] gerade als IndieBand, (S: Naja.) da kann man sich als Mischer endlich mal was wagen.

S: Ja, richtig, richtig.

F: Oder? Das hörst du nicht allzu häufig. [...]

S: Na mein Gefühl war, dass es einfach [...] zu viel wird. [...] [D] as ist jetzt schon sehr, sehr, sehr, also die Songs sind jetzt schon sehr, sehr... weiß nicht, wie ich es nennen soll...

Die Argumentation des Schlagzeugers vollzog sich am Ende anhand der Kombination von Songwriting und Sounderzeugung bzw. -bearbeitung, die einander bedingen bzw. beeinflussen. Zusätzlich von Gewicht war jedoch das angespannte Verhältnis zwischen dem Produzenten und der Band. Erstgenannter hatte nur widerwillig das zusätzliche Drum-Recording genehmigt und galt generell als teilweise schwierig im Umgang, stellte der Band aber in Form seiner Expertise und seines eigenen Tonstudios Ressourcen zur Verfügung, die für diese sonst nicht zugänglich waren. Dementsprechend waren es nicht allein die klanglichen Bedenken des Schlagzeugers, sondern auch die Sicherung der Beziehung zum Produzenten, die hier strukturierend und die klanglichen Aspekte der Selbstpositionierung als >Indie-Band überschreibend in die Klangkonfiguration eingriffen: Die Idee des Tontechnikers ist meines Wissens nach nie ausprobiert worden.

\section{Möglichkeiten der Datenerhebung bei der Suche nach dem srichtigen Sound}

Würde man solche Entstehungsprozesse musikanalytisch begleiten wollen, etwa mit dem Ziel, die Veränderung von Komposition und Klang entlang selbstgewählter und -konstruierter Positionen der beteiligten Akteur*innen und ihrer Interaktionen zu verfolgen, könnte dies mittels der verschiedenen Stadien einer Produktion in Kombination mit derartigen Beobachtungen geschehen, sofern die Audio- bzw. Projektdateien verfügbar wären. Dies ist aber nicht ohne Schwierigkeiten, weil zwecks Übersichtlichkeit in Produktionsprozessen schlecht bewertete Takes oder Einstellungen meistens gelöscht oder überarbeitet werden. Die gröbste Unterteilung der verschiedenen Produktionssta-

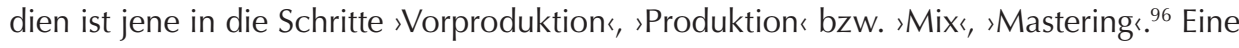
feinere und mit der Praxis einiger Produzent*innen vermutlich kongruente Option wäre das Nachvollziehen anhand der Ist-Zustände eines Songs nach verschiedenen Sessions, die zwecks Absicherung und/oder Reversibilität mit entsprechendem Datum bzw. als gesonderte Versionen abgespeichert werden. ${ }^{97}$ Die Analyse der Kombination von kompositorischen und soundbezogenen Aspekten könnte als eine Lesart - jene der Erzeugenden - der musikwissenschaftlich-analytischen Lesart gegenübergestellt werden, um beispielsweise herauszufinden, inwieweit bestimmte Hörgewohnheiten und -erwartungen sowie Selbstverortungen das Resultat strukturieren, ohne dass dabei im Tonstudio explizit musiktheoretisch oder soundanalytisch vorgegangen wird, zugleich aber bestimmte

96 Huschner 2016, 275.

97 Huschner 2016, Anhang 7. 
Vorstellungen implizit solche Faktoren berücksichtigen.$^{98}$ Oder anders formuliert: Durch eine derartige Gegenüberstellung könnte man musikwissenschaftliche Lesart, künstlerisch-produzierende Intention und die jeweils gewählten Bezugspunkte ${ }^{99}$ abgleichen, womit der soziokulturelle Hintergrund der Klangentstehung zumindest in dieser Hinsicht berücksichtigt würde. Allerdings ist die adäquate Darstellung klanglicher Entwicklungen noch immer problematisch. Man kann lediglich auf die Kombination von Verbalisierung und verfügbarem Material zum Hören hoffen, wie das Beispiel Sex on Fire (2008) der Kings of Leon verdeutlicht: Der Bassist der Band spielte gerne in höheren Lagen und um das klangliche Erscheinungsbild trotzdem konkurrenzfähig zu gestalten, wurde eine Dbx Boombox, ein sub-harmonischer Synthesizer, der eigentlich als Hörequipment vermutlich vor allem für Diskotheken - vorgesehen war ${ }^{100}$, zur Erzeugung zusätzlicher tiefer Frequenzen vor die DI-Box geschaltet. ${ }^{101}$ Erneut ist hier die Idee der klanglichen Konkurrenzfähigkeit anzutreffen, bei der bestimmte Frequenzen im Erscheinungsbild präsent sein sollten, um Hörerwartungen und Ansprüchen der Hörer*innen im Vergleich zu anderen Angeboten des Feldes zu entsprechen. Spannend wäre die Frage, ob diese Veränderung im Studio verhandelt oder einfach mit Verweis auf die Notwendigkeit eines ausgewogenen Frequenzbildes von der Band akzeptiert wurde. Immerhin sind in dem Song deutliche Elemente des Live-Bezugs gegeben, etwa das sich ändernde Tempo, obwohl die veröffentlichte Klangkonfiguration deutlich von einer möglichen Live-Situation abweicht, z.B. aufgrund des sich ändernden Sounds der Bass Drum im Songverlauf. Gleiches dürfte für den mit der Boombox gefundenen Kunstgriff gelten, der live vermutlich nicht zum Einsatz kommen würde. Leider sind auch hier die Informationen mangels wissenschaftlichen Interesses des Bandmanagements nachträglich nicht verfügbar. ${ }^{102}$

Solche durch den Einfluss von Tonstudios gegenwärtig immer vorhandenen Diskrepanzen zwischen gespieltem und komponiertem sowie dann gehörtem Klangmaterial ${ }^{103}$ sollten in einer analytischen Auseinandersetzung mit Klangkonfigurationen der Popmusik nicht unberücksichtigt bleiben, wenn auch ihre Darstellung schwierig ist. Auditiv könnte man solche Prozesse hingegen simpel nachvollziehen: Der Unterschied zwischen einem Schlagzeug ohne Effekte und demselben Schlagzeug mit Effekten ist eindeutig zu hören. Aufgrund dieser unnatürlichen Klangbilder spricht Zagorski-Thomas also berechtigterweise von »sonic cartoons", die aber trotz ihrer Abweichung vom »real thing «104 des Live-Musizierens notwendig sind, um im Feld der Popmusikproduktion zumindest eine kleine Chance auf Profite zu haben.

98 Vgl. Zagorski-Thomas 2015, 119, $129 \mathrm{f}$.

99 Vgl. Porcello 1996, 140-145; Covach 2003, 179f.; die Diskrepanz zwischen Intention und Rezeption thematisiert auch Zagorski-Thomas 2012, $144 \mathrm{f}$.

100 Papenburg 2016.

101 Zagorski-Thomas 2015, 123, Fußnote 14.

102 Ebd., 118-120, $129 \mathrm{f}$.

103 Zu den Unterschieden durch Klangbearbeitung vgl. Huschner 2016, $282 \mathrm{f}$.

104 Zagorski-Thomas 2014, 47, wobei Zagorski-Thomas auch feststellt, dass der Bewertungsmaßstab zunehmend die Aufnahme, nicht mehr die Live-Performance sei (ebd., 201). 


\section{Wandlungsprozesse von Klangkonfigurationen}

Zumindest zwei Song-Beispiele seien hier in Bezug auf klangliche Veränderungen im Verlauf des Produktionsprozesses vorgestellt. Die Klangbeispiele sind zu den jeweiligen Stationen Vorproduktion bzw. Demo, Produktion bzw. Mix und Mastering festgehalten worden.

Die Unterschiede zwischen Demo (Audiobsp. 1) und fertigem Mix (Audiobsp. 2) sind eindeutig hörbar und berühren u.a. jenes Konzept, das Zagorski-Thomas als »clarity « ${ }^{105}$ und ich als »Transparenz «106 bezeichne; in diesen und den folgenden Hörbeispielen geht es um eine eindeutige und detaillierte Wahrnehmung des klanglichen Geschehens. Darüber hinaus erfolgten bei Movie während des Masteringprozesses Bearbeitungen orientiert an einem konkreten, vom Produzenten vorgeschlagenen Bezugspunkt ${ }^{107}$ (Audiobsp. 3): Es wurden ältere Songs der Band Muse ausgewählt, die etwa im Bereich um zwei kHz besonders verstärkt wurden. Eine vergleichbare Bearbeitung führte aber durch die hohen Streicher zu einem unangenehmen Hörempfinden, weswegen auf eine recht abenteuerliche Weise um diesen Bereich herum Anhebungen vorgenommen wurden. Weiterhin entschieden sich die Beteiligten für ein besonders breites Stereobild und gegen ein besonders präsentes Schlagzeug, um sich klanglich von dem gewählten Bezugspunkt Muse abzusetzen, sowie für eine im Vergleich zu den Muse-Songs geringere Kompression.

D)) http://storage.gmth.de/zgmth/media/900/Huschner_Pop_Audio01.mp3

Audiobeispiel 1: Movie - Demo

D)) http://storage.gmth.de/zgmth/media/900/Huschner_Pop_Audio02.mp3

Audiobeispiel 2: Movie - Mix

D)) http://storage.gmth.de/zgmth/media/900/Huschner_Pop_Audio03.mp3

Audiobeispiel 3: Movie - Master

Auch bei der im Folgenden besprochenen Klavierballade sind die Unterschiede zwischen vorproduzierter Demoversion und fertigem Mix klar zu hören (Audiobsp. 4 und 5), weil wie im ersten Beispiel die Tonspuren ausschließlich in einem akustisch nicht optimierten Homestudio und vor allem mit Software-Simulationen von Instrumenten bzw. Verstärkern aufgenommen wurden. Demgegenüber wirken die im Studio aufgenommenen Spuren schon allein wegen des höherwertigen Equipments und dem Wissen des Produzenten bezüglich klanglich-technischer Verbesserungen, z.B. durch EQs, brillanter und in ihrer Kombination transparenter.

105 Zagorski-Thomas 2014, 216-218.

106 Huschner 2016, 189, 283, 294, 298, 299, 312 und passim.

107 Die Mastering-Session ist in meiner Arbeit ausführlich nachzulesen, ich konzentriere mich daher auf ausgewählte Aspekte. Huschner 2016, Anhang 145-176. 
D)) http://storage.gmth.de/zgmth/media/900/Huschner_Pop_Audio04.mp3

Audiobeispiel 4: Klavierballade - Demo

(2)) http://storage.gmth.de/zgmth/media/900/Huschner_Pop_Audio05.mp3

Audiobeispiel 5: Klavierballade - Mix

Bei der Klavierballade diente während des Masterings (Audiobsp. 6) All of Me (2013) von John Legend aufgrund dessen damaligen Erfolges als Orientierungspunkt bezüglich des Klavier- und Vokalklangs. Zugleich musste der Song im Stereobild etwas verengt werden, weil das Klavier sonst breiter und lauter gewirkt hätte als die eigentlich druckvolleren und daher größer gedachten Gitarrensongs des Albums, wie der Produzent anmerkte:

Hier hab' ich z.B. das ganze Stereobild rausgenommen [...]. Weil der Flügel wieder links/rechts sehr weit ist, die Gitarre, da brauchen wir nichts zu machen. [...] Das [...] wirkt [...] natürlich viel lauter als die anderen [Gitarrensongs]. Deswegen hab' ich auch irre wenig Kompression raufgegeben, also relativ wenig halt.

(D)) http://storage.gmth.de/zgmth/media/900/Huschner_Pop_Audio06.mp3

Audiobeispiel 6: Klavierballade - Master

Die oben erläuterten Konzepte der klanglichen Konkurrenz- und Anschlussfähigkeit beziehen sich also nicht nur auf Aspekte der Klangkonfigurationen, sondern können auch innerhalb der eigenen Publikationen maßgeblich sein, um z.B. den Rezipient*innen eine kohärente Projektionsfläche für bestimmte Lesarten zu bieten. Allerdings gilt das zuvor genannte Problem der Konstruktion der Hörenden bzw. eines Textes hier ebenso: So sehr sich Produzent*innen und Musiker*innen auch Gedanken über die Anschluss- und Konkurrenzfähigkeit ihrer Erzeugnisse machen, ist aus diversen, von ihnen nicht kontrollierbaren Gründen die Rezeption von Seiten der Hörenden nicht zu prädeterminieren. Sicherlich können Hörgewohnheiten und Klangpräferenzen in Form von erfolgreichen Titeln aufgegriffen und weitgehend implementiert werden. Allerdings arbeiten nahezu alle professionellen Studioakteur*innen auf diese Weise, wodurch eine große Masse an vergleichbar gut und ähnlich klingenden Klangkonfigurationen entsteht, von denen einige wenige dann aus - vorwiegend nicht smusikalisch-klanglichen Gründen - kommerziell erfolgreich werden oder eben nicht. Inwiefern die innerhalb der Studios ausgehandelten Lesarten außerhalb der Studios überhaupt transportiert werden können bzw. Entsprechung in der Rezeption finden, wäre unabhängig davon zu überprüfen.

\section{POPMUSIK: ANALYSE UND PRODUKTION}

Man müsste also die oben genannten Perspektiven der Musikwissenschaft und der vor allem durch technische Faktoren beeinflussten Studioakteur*innen um die der Hörenden ergänzen. Während Moores »sound-box« und Zagorski-Thomas' Musicology of Record 
Production das Tonstudio bereits in die Popmusikanalyse einbezogen haben, möchte ich mit der Analyse der Interaktionen zwischen den dort tätigen Akteur*innen einen Schritt weitergehen, um herauszufinden, warum beispielsweise die »sound-box" eines bestimmten Songs auf eine bestimmte Art und Weise und nicht anders konfiguriert ist, und welche verschiedenen Stadien diese »sound-box" aus welchen Gründen durchlaufen hat. Gleiches gilt für die Vielzahl von zutreffenden Beobachtungen bezüglich des Einflusses der Studiointeraktionen auf die Klangkonfigurationen bei Zagorski-Thomas, was angesichts seiner langjährigen Tätigkeit in diesem Bereich nicht verwunderlich ist. Es geht aber nicht nur darum festzustellen, dass diese Bearbeitungen von Klang vorgenommen wurden, sondern auch entlang welcher Lesarten und Zielstellungen diese vollzogen werden; im Prinzip sind damit genau die Prozesse thematisiert, die Plodroch anhand von Songwriting Camps beschreibt und begleitet, ohne aber Zugriff auf Klangmaterial zu erhalten. ${ }^{108}$

Sinnvoll ist eine solche Ausweitung der Perspektive schon deshalb, weil durch die Studioarbeit eine Übereinstimmung hinsichtlich des Strebens nach Differenzierung bei Produzierenden und Konsument*innen zwar potentiell angelegt ist ${ }^{109}$, die dazu genutzten Eigenschaften - handelt es sich um klangliche, musikalische oder ganz andere Faktoren? - aber völlig offen sind: Wenn die Strukturierung des Hörerlebnisses z.B. in einem bestimmten Part durch den Einsatz eines Subbasses unterstützt oder vollzogen wird, Hörende den Song aber über Kopfhörer oder Handy-Lautsprecher mit eingeschränktem Frequenzspektrum rezipieren, kann diese Idee bzw. die Intention der Künstler*innen oder Produzierenden nicht greifen. ${ }^{10}$ Wenn ein Song Größe und Weite durch ein überbreites Stereobild oder Hallräume transportieren soll und über einen Mono-Lautsprecher abgespielt wird, kann dieser Effekt ebenfalls nicht in Erscheinung treten. ${ }^{11}$ Dabei geht es nicht um ein >richtiges`, ‘falsches` oder sideales` Hören von populärer Musik ${ }^{112}$, sondern um das Problem der wechselnden Nutzungskontexte sowie der damit einhergehenden Wiedergabebedingungen, die bestimmte Lesarten zumindest temporär inadäquat werden lassen und mit denen sich auch die Akteur*innen in den Tonstudios auseinandersetzen. ${ }^{13}$ Ferner würden mit einer solchen Methodik genau die Prozesse untersucht werden, die jene von Moore angenommenen Interpretationsbereiche erst erzeugen.

108 Plodroch 2017.

109 Bourdieu 2001, 259-263; Bourdieu 2012, 367-373.

110 Diese Schwierigkeiten betreffen nicht nur die Popmusik, haben aber durch das grundsätzliche Ziel der massenhaften Konsumtion in diesem Feld eine ganz andere Tragweite: Wenn die im Studio großartig klingenden Klangkonfigurationen durch Handy-Lautsprecher, Küchen- oder Autoradios und Kopfhörer nicht trotzdem ansprechend klingen, ist ihre Anschlussfähigkeit für einen großen Teil der potentiellen Hörer*innen nicht gegeben, sie werden nicht in ausreichender Form zirkulieren und nicht ausreichend Profite erwirtschaften. Erneut zeigt sich bei solchen Überlegungen der Einfluss technischer und ökonomischer Faktoren, die nach Möglichkeit bei der Analyse berücksichtigt werden sollten.

111 Huschner 2016, 324f., Anhang 156.

112 Vgl. Doehring 2012, 30.

113 Zagorski-Thomas 2014, 77. 
Darüber hinaus könnte durch die Gegenüberstellung dreier Perspektiven - Klangkonfigurationen, Produktion, Rezeption - das Problem gelöst werden, dass Hörende oftmals gar nicht genau oder nicht präzise genug beschreiben können, was an bestimmten Songs Wohlgefallen und/oder die Nutzung als Differenzierungsgegenstand auslöst. Im Zusammenhang mit den Informationen einer Produktionssession könnten solche Aussagen wahrscheinlich genauer zugeordnet und gleichzeitig z.B. dahingehend untersucht werden, inwieweit und ob überhaupt die Kommunikation zwischen Künstler*innen, Produzierenden und Hörenden den jeweiligen Vorstellungen entspricht, welche Veränderungen der Deutung bei der Aufschlüsselung der Urheberschaft zu erwarten wären, inwieweit technische Voraussetzungen die Erzeugung und Rezeption verändern, welche soziokulturellen Hintergründe die Intention bzw. Rezeption betreffen und Ähnliches.

Das beseitigt nicht das Problem der weitgehenden Irrelevanz des Wollens und der Entscheidungen von Akteur*innen in Tonstudios, wenn es um kommerziellen Erfolg und qualitative Bewertungen außerhalb des Tonstudios geht, kann aber einen Weg aufzeigen, wie man analytisch einer Musikform begegnet, die auf die Nutzung des Tonstudios ${ }^{114}$ und die Generierung von möglichst neuartigen und zugleich soziokulturell anschlussfähigen Klängen ausgerichtet ist. Natürlich wäre der dafür zu betreibende Aufwand immens und das Problem der adäquaten Darstellung oder Wiedergabe von Soundveränderungen wie in den oben hinzugezogenen Klangbeispielen wäre noch immer nicht gelöst. Der Mehrwert aber, der sich aus der Einbeziehung der Produktionsperspektive insgesamt ergibt, und die daraus resultierenden Fragen bezüglich der Funktionsweise des Feldes der populären Musikproduktion sollten in einer integrativen und adäquaten Analyse von Popmusik stärker als bisher berücksichtigt werden.

\section{Literatur}

Appen, Ralf von (2015), „Ear Candy: What Makes Ke\$ha's `Tik Tok« Tick?«, in: Song Interpretation in 21th-Century Pop Music, hg. von Ralf von Appen, André Doehring, Dietrich Helms und Allan F. Moore, Farnham: Ashgate, 29-51.

Appen, Ralf von / André Doehring / Dietrich Helms / Allan F. Moore (Hg.) (2015), Song Interpretation in 21th-Century Pop Music, Farnham: Ashgate.

Barber, Simon (2012), „Soundstream: The Introduction of Commercial Digital Recording in the United States", in: Journal on the Art of Record Production 7. http://www.arpjournal.com/asarpwp/soundstream-the-introduction-of-commercial-digital-recording-in-the-united-states (13.08.2018)

Bennett, Samantha (2009), »Revolution Sacrilege! Examining the Technological Divide among Record Producers in the late 1980s«, Journal on the Art of Record Production 4. http://www.arpjournal.com/asarpwp/revolution-sacrilege-examining-the-technological-divide-among-record-producers-in-the-late-1980s (13.08.2018) 
Böhmermann, Jan (2017), Eier aus Stahl. Max Giesinger und die deutsche Musikindustrie, Neo Magazin Royale, 6.4.2017. https://www.zdf.de/comedy/neo-magazin-mit-janboehmermann/eier-aus-stahl-max-giesinger-100.html (17.4.2017)

Bourdieu, Pierre (1993a), Soziologische Fragen, Frankfurt a.M.: Suhrkamp.

— (1993b), „Über Ursprung und Entwicklung der Arten der Musikliebhaber«, in: ders., Soziologische Fragen, Frankfurt a.M.: Suhrkamp, 147-152.

(2001), Die Regeln der Kunst, Genese und Struktur des literarischen Feldes, Frankfurt a.M.: Suhrkamp.

(2005), „Ökonomisches Kapital - Kulturelles Kapital - Soziales Kapital», in: ders., Die verborgenen Mechanismen der Macht (= Schriften zur Politik und Kultur, Bd. 1), Hamburg: VSA, 49-79.

- (2012), Die feinen Unterschiede. Kritik der gesellschaftlichen Urteilskraft, Frankfurt a.M.: Suhrkamp.

Bundesverband Musikindustrie (2017), Musikindustrie in Zahlen 2016. http://www.musikindustrie.de/fileadmin/bvmi/upload/02_Markt-Bestseller/MiZ-Grafiken/2016/bvmi2016-musikindustrie-in-zahlen-jahrbuch-ePaper_final.pdf (17.4.2017)

Burgess, Richard James (2005), The Art of Music Production, London: Omnibus Press.

- (2011), "Interview with Lauren Christy of The Matrix«, Journal on the Art of Record Production 5. http://www.arpjournal.com/asarpwp/interview-with-lauren-christy-ofthe-matrix (13.08.2018)

Burns, Gary (1987), »A Typology of `Hooks` in Popular Records«, Popular Music 6/1, $1-20$.

Covach, John (2003), „Pangs of History in late 1970s New-Wave Rock», in: Analyzing Popular Music, hg. von Allan F. Moore, Cambridge: Cambridge University Press, 173-195.

Danielsen, Anne (2015), »Metrical Ambiguity or Microrhythmic Flexibility? Analysing Groove in Nasty Girl by Destiny's Child", in: Song Interpretation in 21th-Century Pop Music, hg. von Ralf von Appen, André Doehring, Dietrich Helms und Allan F. Moore, Farnham: Ashgate, 53-71.

De Clercq, Trevor / David Temperley (2001), „A Corpus Analysis of Rock Harmony«, Popular Music 30/1, 47-70.

Dockwray, Ruth / Allan F. Moore (2010), »Configuring the Sound-Box 1965-1972«, Popular Music 29/2, 181-197.

Doehring, André (2012), »Probleme, Aufgaben und Ziele der Analyse populärer Musik«, in: Black Box Pop. Analysen Populärer Musik (= Beiträge zur Popularmusikforschung, Bd. 38), hg. von Dietrich Helms und Thomas Phleps, Bielefeld: transcript, 23-42. (2015), »Andrés's >New For U«: New for Us, On Analysing Electronic Dance Music«, in: Song Interpretation in 21th-Century Pop Music, hg. von Ralf von Appen, André Doehring, Dietrich Helms und Allan F. Moore, Farnham: Ashgate, 133-155.

Doyle, Peter (2009), »Working for the Man. Figuring the Artist-Producer Relationship«. Podcasts of Conferences, Seminars and Events hosted by the School of English, Com- 
munications and Performance Studies, Monash University. http://www.digitalpodcast.com/items/7696234 (16.4.2017)

Elsdon, Peter (2016), „Review. The Musicology of Record Production«, iaspm@journal 6/2, 177-180.

Everett, Walter (1999), The Beatles As Musicians. Revolver through the Anthology, New York: Oxford University Press.

(2001), The Beatles as Musicians. The Quarry Men through Rubber Soul, New York: Oxford University Press.

- (2015), »Death Cab for Cutie's >l Will Follow You Into The Darkı as Exemplar of Conventional Tonal Behavior in Recent Rock Music«, in: Song Interpretation in 21th-Century Pop Music, hg. von Ralf von Appen, André Doehring, Dietrich Helms und Allan F. Moore, Farnham: Ashgate, 9-28.

Foucault, Michel (2005), Analytik der Macht, Frankfurt a.M.: Suhrkamp.

— (2013), Archäologie des Wissens, Frankfurt a.M.: Suhrkamp.

Frith, Simon (1998), Performing Rites: On the Value of Popular Music, Oxford: Oxford University Press.

— (2012), »The Place of the Producer in the Discourse of Rock«, in: The Art of Record Production: An Introductory Reader to a New Academic Field, hg. von Simon Zagorski-Thomas und Simon Frith, Farnham: Ashgate, 207-222.

Hampp, Andrew (2012), »Alex Clare Talks Microsoft Ad, Amy Winehouse \& Career ReStart«. http://www.billboard.com/articles/columns/the-juice/495155/alex-clare-talksmicrosoft-ad-amy-winehouse-career-re-start (28.5.2017)

Hennion, Antoine (1981), Les Professionnels du Disque. Une sociologie des variétés, Paris: Métaillié.

_ (1983), »The Production of Success: An Anti-Musicology of the Pop Song«, Popular Music 3, 159-193.

_ (1989), „An Intermediary between Production and Consumption: The Producer of Popular Music«, Science, Technology \& Human Values 14/4, 400-424.

Hodgson, Jay (2010), »A Field Guide to Equalisation and Dynamics Processing on Rock and Electronica Records«, Popular Music 29/2, 283-297.

Howlett, Mike (2012), »The Record Producer As Nexus«, Journal on the Art of Record Production 6, http://www.arpjournal.com/asarpwp/the-record-producer-as-nexus (13.8.2018)

Huschner, Roland (2016), „[...] if it would be me producing the song..." Eine Studie zu den Prozessen in Tonstudios der populären Musikproduktion, Phil. Diss. Humboldt-Universität zu Berlin. http://edoc.hu-berlin.de/dissertationen/huschner-roland-2016-07-18/PDF/huschner.pdf (28.5.2016)

Keister, Jay / Jeremy L. Smith (2008), »Musical ambition, cultural accreditation and the nasty side of progressive rock«, Popular Music 17/3, 433-455. 
Kramarz, Volkmar (2014), Warum Hits Hits werden. Erfolgsfaktoren der Popmusik. Eine Untersuchung erfolgreicher Songs und exemplarischer Eigenproduktionen, Bielefeld: transcript.

Latour, Bruno (2010), Eine neue Soziologie für eine neue Gesellschaft. Einführung in die Akteur-Netzwerk-Theorie, Frankfurt a.M.: Suhrkamp.

Massey, Howard (2000), Behind the Glass. Top Record Producers Tell How They Craft The Hits, San Francisco: Miller Freeman Books.

Meintjes, Louise (2003), Sound of Africa! Making Music Zulu in a South African Studio, Durham: Duke University Press.

Moehn, Frederick J. (2005), »The Disc is not the Avenues: Schismogenetic Mimesis in Samba Recording", in: Wired for Sound. Engineering and Technologies in Sonic Cultures, hg. von Paul D. Greene und Thomas Porcello, Middleton: Wesleyan University Press, 47-83.

Morrow, Guy (2012), „Creative Conflict In A Nashville Studio: A Case Of Boy \& Bear«, Journal on the Art of Record Production 6. http://arpjournal.com/asarpwp/creativeconflict-in-a-nashville-studio-a-case-of-boy-bear (13.08.2018)

Moore, Allan F. (2001), Rock. The Primary Text. Developing a Musicology of Rock [1993], 2. Auflage, Aldershot: Ashgate.

_ (2002), »Authenticity as Authentification«, Popular Music 21/2, 209-223. (2012), Song Means: Analysing and Interpreting Recorded Popular Song, Farnham: Ashgate.

Papenburg, Jens Gerrit (2008), „Stop/Start Making Sense! Ein Ausblick auf Musikanalyse in Popular Music Studies und technischer Medienwissenschaft», in: Sound Studies. Traditionen - Methoden - Desiderate, hg. von Holger Schulze, Bielefeld: transcript, 91-108.

- (2016), „Enhanced Bass: On 1970 Disco Culture's Listening Devices», in: Sound as Popular Culture. A Research Companion, hg. von Jens Gerrit Papenburg und Holger Schulze, Cambridge: MIT Press, 205-214.

Plodroch, Ina (2017), „Die Pop-Fabrik. So werden Hits gemacht«, Deutschlandfunk, Corso-Spezial, 17.4.2017, http://www.deutschlandfunk.de/die-pop-fabrik-so-werdenhits-gemacht.807.de.html?dram\%3Aarticle_id=383749 (18.4.2017)

Porcello, Thomas (1996), Sonic Artistry: Music, Discourse, and Technology in the Sound Recording Studio, Ph.D., University of Texas at Austin.

Riedemann, Frank (2012), „Computergestützte Analyse und Hit-Songwriting«, in: Black Box Pop. Analysen Populärer Musik (= Beiträge zur Popularmusikforschung, Bd. 38), hg. von Dietrich Helms und Thomas Phleps, Bielefeld: transcript, 43-56.

Spicer, Mark S. (2000), »Large Scale Strategy and Compositional Design in the Early Music of Genesis", in: Expression in Pop-Rock Music. A Collection of Critical and Analytical Essays, hg. von Walter Everett, New York: Taylor and Francis, 77-111.

Steinbrecher, Bernhard (2016), Das Klanggeschehen in populärer Musik. Perspektiven einer systematischen Analyse und Interpretation, Köln: Böhlau. 
Tagg, Philip (2000), »The Work«: An Evaluative Charge«, in: The Musical Work: Reality or Invention?, hg. von Michael Talbot, Liverpool: Liverpool University Press, 153-167.

Thompson, Paul / Lashua, Brett (2016), »Producing Music, Producing Myth? Creativity in Recording Studios«, iaspm@journal 6/2, 70-90. http://www.iaspmjournal.net/ index.php/IASPM_Journal/article/view/775/pdf (28.5.2017)

Weinstein, Gregory (2016), »Air Flows: Breath, Voice, and Authenticity in Three Recordings«, iaspm@journal 6/2, 117-138. http://www.iaspmjournal.net/index.php/ IASPM_Journal/article/view/780/pdf (28.5.2017)

Wicke, Peter (1993), „Popmusik als Industrieprodukt», in: ders., Vom Umgang mit Popmusik, Berlin: Volk und Wissen. http://www2.hu-berlin.de/fpm/textpool/texte/ wicke_popmusik-als-industrieprodukt.htm (28.5.2017)

_ (2003), »Popmusik in der Analyse«, Acta Musicologica 75/1, 107-126. http:// www2.hu-berlin.de/fpm/textpool/texte/wicke_popmusik-in-der-analyse.htm (30.4.2017)

- (2004), „Über die diskursive Formation musikalischer Praxis. Diskurs-Strategien auf dem Feld der populären Musik«, in: Festschrift. Prof. Dr. Gerd Rienäcker zum 65. Geburtstag, hg. von Stephan Aderhold, Berlin. http://festschrift65-rienaecker.de/018Wicke\%20S.\%20163-174.PDF (28.5.2017)

— (2016), »The Sonic«, in: Sound as Popular Culture. A Research Companion, hg. von Jens Gerrit Papenburg und Holger Schulze, Cambridge: MIT Press, 23-30.

Zagorski-Thomas, Simon (2010), »The Stadium in your Bedroom: Functional Staging, Authenticity and the Audience-led Aesthetic in Record Production«, Popular Music 29/2, 251-266.

- (2012), „Musical Meaning and the Musicology of Record Production«, in: Black Box Pop. Analysen Populärer Musik (= Beiträge zur Popularmusikforschung, Bd. 38), hg. von Dietrich Helms und Thomas Phleps, Bielefeld: transcript, 135-148.

(2014), The Musicology of Record Production, Cambridge: Cambridge University Press.

(2015), »An Analysis of Space, Gesture and Interaction in Kings of Leons's ıSex on Fire«", in: Song Interpretation in 21th-Century Pop Music, hg. von Ralf von Appen, André Doehring, Dietrich Helms und Allan F. Moore, Farnham: Ashgate, 133-155.

Zak, Albin J. (2001), The Poetics of Rock. Cutting Tracks, Making Records, Berkeley: University of California Press.

\section{Diskographie}

65daysofstatic (2016), No Man's Sky: Music For An Infinite Universe, Laced Music.

Alex Clare (2011), The Lateness Of The Hour, Universal Island Records.

Avril Lavigne (2002), Let Go, Arista Records.

Beach Boys (1967/2001), Smiley Smile (2001 Remaster), Capitol Records 
Bob Dylan (1967), John Wesley Harding, Sony Music.

John Legend (2013), Love In The Future, Columbia Records.

Keane (2004), Hopes and Fears, Universal Island Records.

Ke\$ha (2009), Animal, RCA/Jive Label Group.

Kings of Leon (2008), Only by the Night, RCA.

Lacuna Coil (2002), Comalies, Century Media.

Midge Ure (1996), Breathe, BMG.

Shawn Lee's Ping Pong Orchestra (2006), Voices and Choices, Ubiquity Records.

Sigur Rós (2007), Hvarf - Heim, Parlophone Records.

Soundgarden (1994), Superunknown, A\&M Records.

The xx (2009), XX, Young Turks/XL Recordings.

Tori Amos (1996), Hey Jupiter, Atlantic Records. 